\title{
An 86-year-old man with septic arthritis of the knee
}

\author{
Tasaduq Fazili MD, Calden Sharngoe MD, Waleed Javaid MD
}

\section{CASE PRESENTATION}

An 86-year-old man with a medical history significant for an aortic valve replacement and pacemaker insertion $>20$ years previously developed a stress fracture of the left leg, which was placed in an immobilizer cast. One week later, he developed progressive left knee swelling and was admitted to the hospital. A physical examination was significant only for a grade 2 (of 6) systolic murmur over the aortic area, and mild swelling and tenderness of the left knee. The surrounding skin was not erythematous. He exhibited leukocytosis with a white blood cell count of $15.1 \times 10^{9} / \mathrm{L}$. An aspirate of joint fluid from the left knee revealed a white blood cell count of $12.9 \times 10^{9} / \mathrm{L}$ with $82 \%$ neutrophils. A Gram-stained smear of the joint fluid revealed many white blood cells with no bacteria. Culture of this aspirate for bacteria and fungi was negative. A bone scan showed increasing uptake in the left tibia, suggestive of osteomyelitis. The patient was discharged on four weeks of ceftriaxone ( $2 \mathrm{~g}$ intravenously once per day) for presumed bacterial septic arthritis/osteomyelitis. He presented three weeks later with increasing left knee pain and swelling. A joint washout of the knee and surgical debridement of the proximal tibia was performed. A Gram stain (Figure 1) and modified acid-fast stain (Figure 2) of the joint fluid are shown.

\section{DIAGNOSIS}

A Gram stain of the synovial fluid revealed branching Gram-positive rods (Figure 1). Modified acid-fast staining revealed partially acid-fast branching filamentous rods (Figure 2). Cultures from the debrided

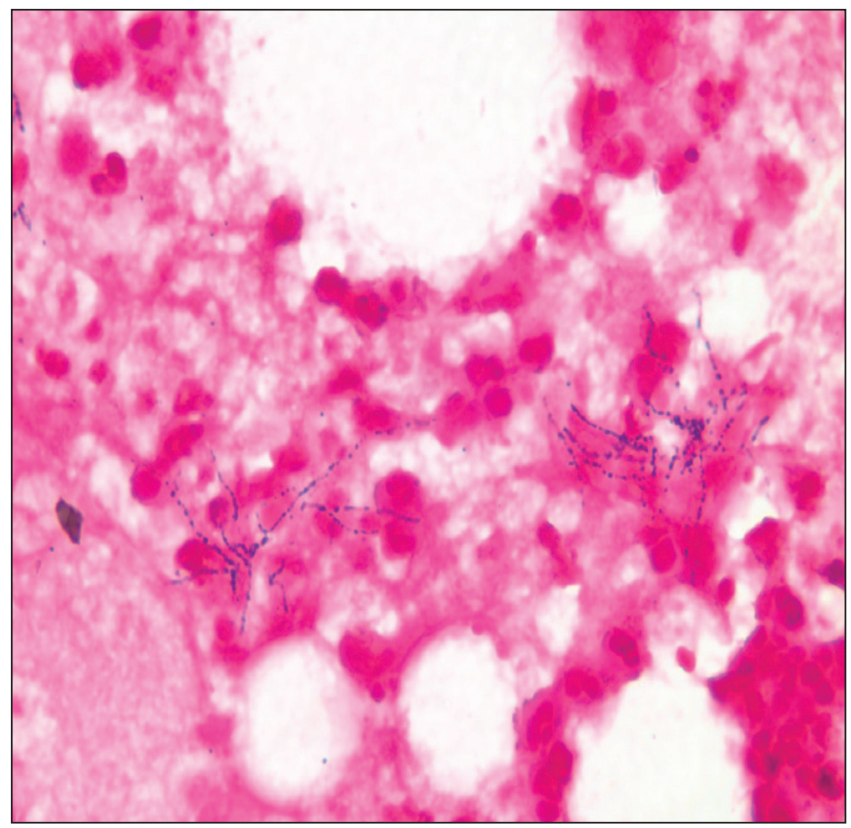

Figure 1) Gram stain of the joint fluid bone and knee joint fluid grew Nocardia pseudobrasiliensis. Species identification was performed using DNA sequencing. The patient was started on intravenous imipenem and amikacin, but continued to worsen, developing foot ischemia requiring a below-knee amputation. Postoperatively, he developed skin and soft tissue abscesses on his back and arm. Cultures from these acscesses also grew $N$ pseudobrasiliensis. Based on susceptibility results, the patient's antibiotic therapy was subsequently switched to trimethoprim/sulfamethoxazole and ciprofloxacin. The patient responded to this therapy, with resolution of his joint and skin and soft tissue infections.

\section{DISCUSSION}

Nocardia species are ubiquitous environmental saprophytes present in soil, organic matter and water. Human infection usually arises from direct inoculation of the skin or soft tissues, or by inhalation (1). Nocardia can have a wide spectrum of presentations, ranging from mild cutaneous disease to aggressive, invasive disease with central nervous system, ocular, pulmonary and joint involvement (1).

$\mathrm{N}$ pseudobrasiliensis was previously identified as Nocardia brasiliensis. However, it was noted that some isolates of $\mathrm{N}$ brasiliensis had different chemical characteristics and disease spectrum (2). In 1996, Ruimy et al (3) suggested a new Nocardia species that caused more invasive disease and exhibited a different antibiotic susceptibility pattern than $N$ brasiliensis, which was named $N$ pseudobrasiliensis.

$\mathrm{N}$ pseudobrasiliensis is associated with invasive disease, mostly in patients with underlying immunosuppression, whereas $N$ brasiliensis typically causes localized cutaneous infections in immunocompetent individuals (4). Isolates of $N$ pseudobrasiliensis are typically susceptible

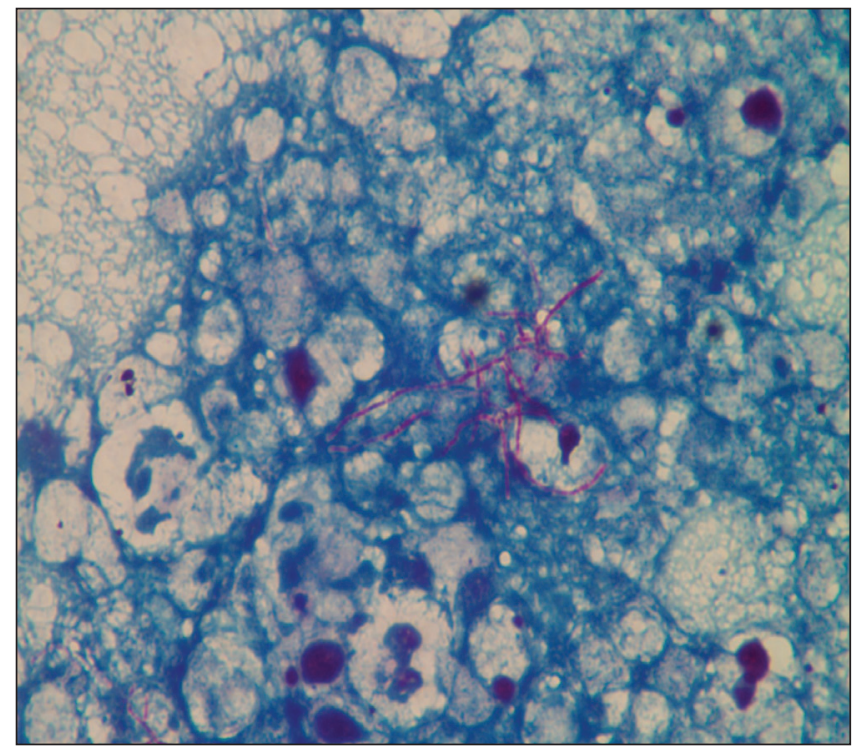

Figure 2) Modified acid-fast stain of the joint fluid

Department of Medicine/Infectious Disease, State University of New York Upstate University, Syracuse, New York, USA Correspondence: Dr Tasaduq Fazili, Department of Medicine/Infectious Disease, State University of New York Upstate Medical University, 725 Irving Avenue, Syracuse, New York 13104, USA. Telephone 315-464-9360, fax 315-464-9361, e-mail fazilit@upstate.edu 
to ciprofloxacin and clarithromycin but are resistant to imipenem, minocycline and amoxicillin-clavulanic acid, whereas the reverse antibiotic susceptibility pattern is typically observed with isolates of $N$ brasiliensis. Both species are generally susceptible to trimethoprim/ sulfamethoxazole and linezolid $(5,6)$.

Diagnosis is suggested by demonstration of non-spore-forming Gram-positive branching rods on Gram stain of tissue. Actinomyces also have similar features on Gram stain, but can be differentiated by modified acid-fast stain. Nocardia are partially acid-fast, unlike Actinomyces. A precise diagnosis can be made by culture of the organism. Nocardia can grow in sheep blood and chocolate agar. However, they grow slowly; hence, cultures may have to be kept for at least a

\section{REFERENCES}

1. Wallace RJ Jr, Brown BA, Blacklock Z. Nocardia species. Mandell, Douglas, and Bennett's Principles and Practice of Infectious Diseases, 7th edn. 2009;254:3199-3206.

2. Wallace RJ Jr, Brown BA, Blacklock Z, et al. New Nocardia taxon among isolates of Nocardia brasiliensis associated with invasive disease. J Clin Microbiol 1995;33:1528-33.

3. Ruimy R, Riegel P, Carlotti A, et al. Nocardia pseudobrasiliensis sp. nov., a new species of Nocardia which groups bacterial strains previously identified as Nocardia brasiliensis and associated with invasive diseases. Int J Syst Bacteriol 1996;46;259-64.

4. Brown BA, Lopes JO, Wilson RW, et al. Disseminated Nocardia pseudobrasiliensis infection in a patient with AIDS in Brazil. Clin Infect Dis 1999;28:144-5. week $(1,7)$. Speciation is performed using DNA sequence analysis and is important in terms of treatment, as outlined in our case.

Treatment of nocardiosis generally requires both antibiotics and surgical intervention, depending on the clinical manifestation (1). Trimethoprim/sulfamethoxazole is generally the first line of treatment, along with another agent as determined by the antibiotic susceptibility pattern (imipenem, amikacin or ciprofloxacin) $(1,5,6)$. Duration of therapy depends on the type of infection. Primary cutaneous infection can be treated with one to three months of therapy, while invasive central nervous system infections need to be treated for as long as six to 12 months. Immunosuppressed patients may require longer than 12 months of therapy (1).

5. Brown-Elliott, Brown JM, Conville PS, Wallace RJ Jr. Clinical and laboratory features of the Nocardia spp. based on current molecular taxonomy. Clin Microbiol Review 2006;19:259-82

6. Lebeaux D, Lanternier F, Degand N, et al. Nocardia pseudobrasiliensis as an emerging cause of opportunistic infection after allogeneic hematopoietic stem cell transplantation. J Clin Microbiol 2010;48:656-59.

7. Smego RA, Harry A. Clinical spectrum of Nocardia brasiliensis infection in the United States. Clin Infect Dis 1984;6:164-80. 


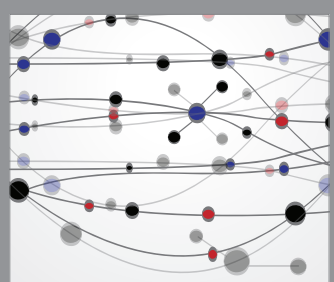

The Scientific World Journal
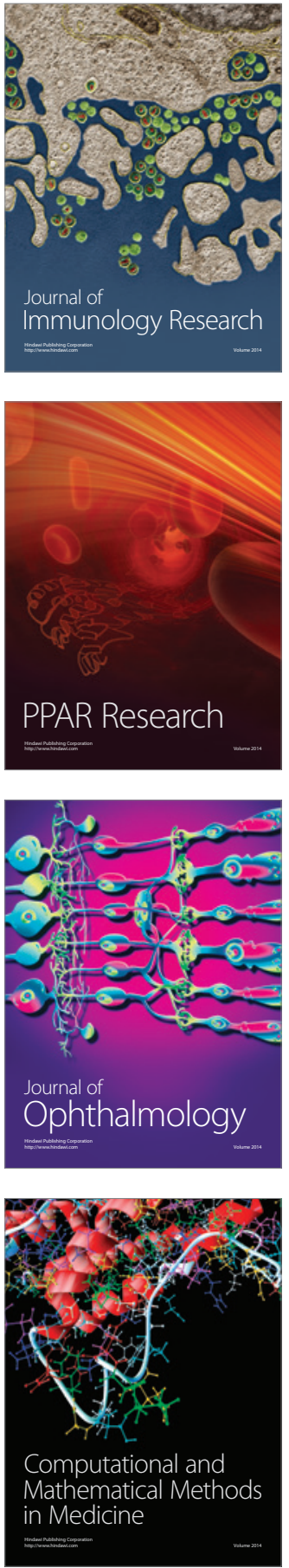

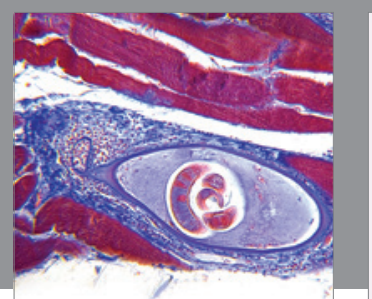

Gastroenterology Research and Practice

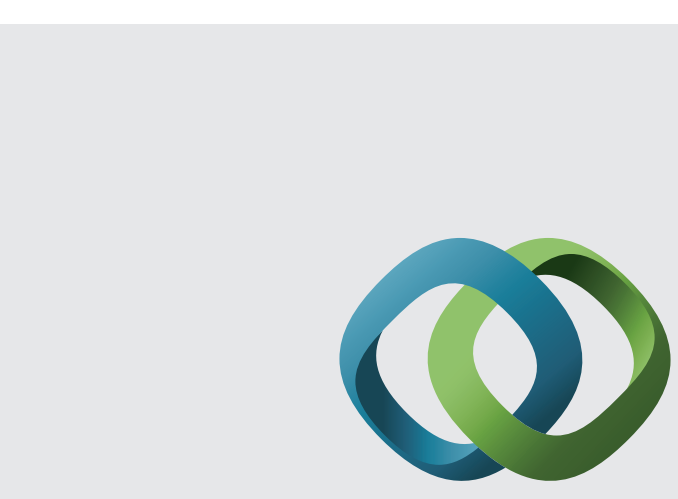

\section{Hindawi}

Submit your manuscripts at

http://www.hindawi.com
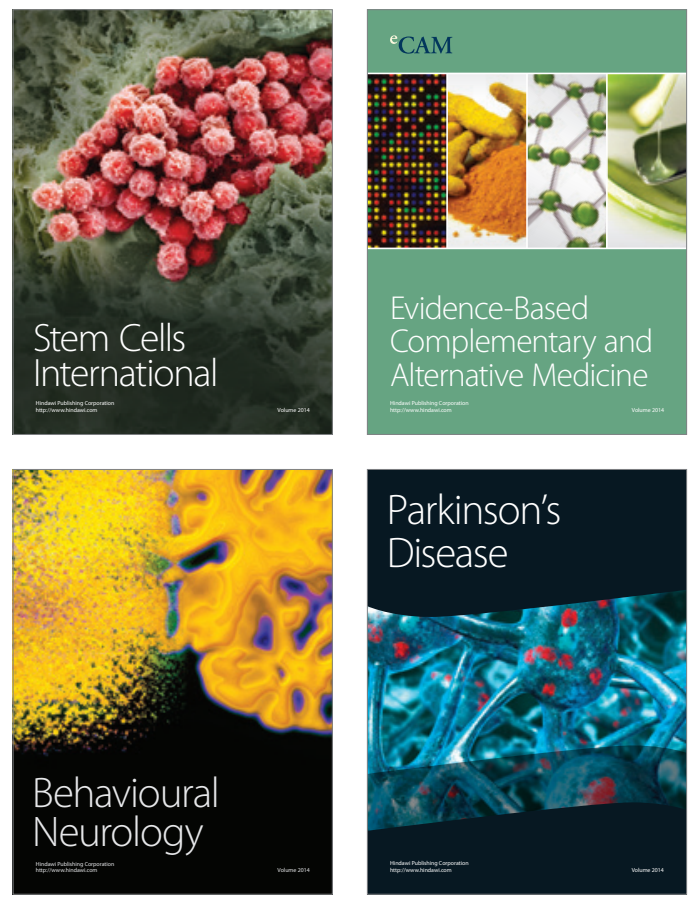
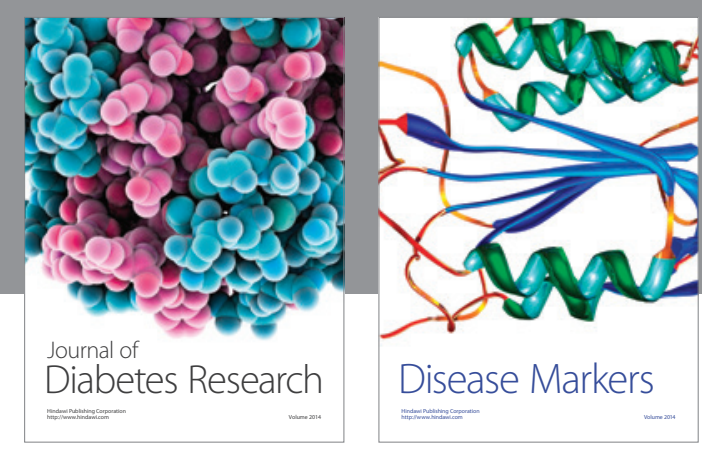

Disease Markers
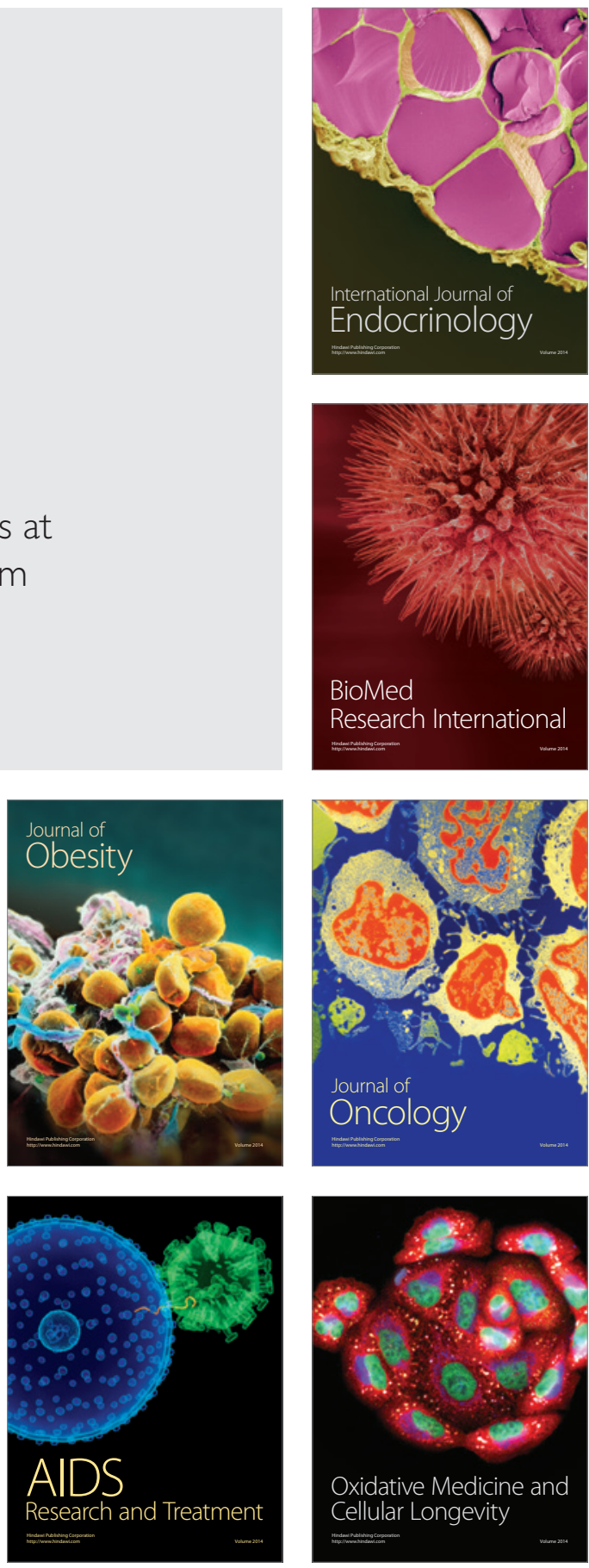